\title{
The role of inflammation in cardiac arrhythmias pathophysiology
}

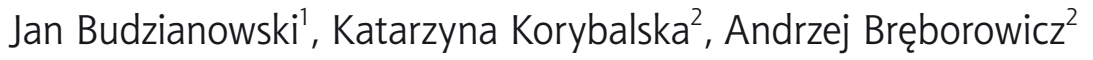 \\ ${ }^{1}$ Department of Cardiology, Hospital, Nowa Sól, Poland \\ ${ }^{2}$ Department of Pathophysiology, Poznan University of Medical Sciences, Poland
}

\begin{abstract}
The pathophysiology of cardiac arrhythmias is highly complex. Inflammation causes electrophysiological changes that contribute to increased vulnerability of arrhythmias, a process known as electrical remodeling. There have been many biomarkers and proteins associated with pathophysiology of cardiac arrhythmias such as C-reactive protein, tumor necrosis factor- $\alpha$, interleukin-2, interleukin- 6 , interleukin-8, and monocyte chemoattractant protein-1. At present large attention is paid to the clinical studies on the inflammation in the pathogenesis of the atrial fibrillation (AF), given the limited effectiveness of the current therapeutic approaches. Understanding the main process, molecular mechanism and signaling pathways underlying the pathogenesis of the inflammatory dilated cardiomyopathy (iDCM) will be essential for developing new effective therapeutic strategies.
\end{abstract}

Keywords: inflammation, atrial fibrillation, biomarkers, remodeling.

\section{Introduction}

There have been numerous clinical studies supporting the important role of inflammation in the pathophysiology of many cardiovascular diseases including: coronary artery disease, heart failure, hypertension, cardiac arrhythmias. These systemic diseases are associated with low-grade inflammation and increased levels of proinflammatory cytokines [1].

Inflammation plays an important role in pathophysiology of atherosclerosis. It is involved in atherosclerosis from its formation and progression to its final stage, i.e. systemic thrombosis [2]. Inflammation is also involved in the initiation and maintenance of atrial fibrillation as well as its thromboembolic complications [3]. The inflammatory system, comprised of specialized cells and mediators, is responsible for complex responses to all forms of injuries. It stimulates inflammatory reactions by activating leukocytes, macrophages, mast cells, fibroblasts, and endothelial cells. Acute inflammation leads to the tissue repair, while the chronic inflam- mation can cause damage and pathological remodeling. Nevertheless, these two dimensions of inflammation may coexist with each other.

The immune system, inflammation and oxidative stress are closely related to common pathological responses. Immune factors (e.g. complement) initiate and propagate the inflammatory reactions. If this reaction is not properly controlled, it can exacerbate the chronic inflammation (e.g. granuloma) [4]. Considering the significance of the inflammatory/immune aspects of cardiac diseases, it needs to be emphasized that inflammation might play an important role in the cardiac cells' function, survival and death. Indeed, inflammatory processes lead to structural and electrical remodeling of the myocardium. Hence, the main pathophysiological mechanisms responsible for the formation of arrhythmias in the cardiac myocardial tissue include ectopic beats, triggered activity and reentry mechanisms. Additionally, inflammation alters electrophysiological properties of the myocardium leading to vari- 
ous forms of arrhythmias: from benign supraventricular ectopic beats to malignant forms of ventricular tachycardias and ventricular fibrillation [6]. At present large attention is paid to the clinical studies on the inflammation in the pathogenesis of the $A F$, given the limited effectiveness of the current therapeutic approaches. In this paper we elaborated pathophysiological aspects of inflammation process in cardiac arrhythmias, emphasized the role of proinflammatory biomarkers involved in atrial fibrillation.

\section{Pathophysiology}

Klein et al, have documented the adverse role of inflammation in the pathogenesis of arrhythmias. They described pathological changes in the membrane potential and heterogeneous impulse conduction due to fibrosis and scarring of the myocardial tissue. They also proposed that the formation of cardiac arrhythmias in the inflamed myocardium occurs due to three factors: structural changes, ventricular dynamics and vascular factors. They further remarked that the parameters of ventricular dynamics such as increased wall tension, increased myocardial oxygen consumption and diminished coronary reserve in case of disturbed systolic or diastolic left ventricular function also contribute to the increased incidence of arrhythmias. It was observed that vascular factors can further increase the arrhythmogenicity of the inflamed myocardium through the disturbance of micro- and macrovascular perfusion and the resulting myocardial ischemia. Hence, myocardial biopsy carried out to detect myocarditis is an important additional examination which can improve the differential diagnosis and treatment of patients with cardiac arrhythmias of obscure etiology [7]. Although the inflammatory process of the myocardium may itself be arrhythmogenic, the change in the electrophysiological properties of the myocardium (i.e. electrical remodeling) may also play a role in cardiac arrhythmias [8].

For example, in experimental autoimmune myocarditis (EAM) in rats, demonstrated the changes in the ventricular electrophysiologic properties and in the level of gene expression of cardiac ion channels. During the acute phase of the myocarditis, the ventricular vulnerability was increased and, as a result, the triggered activity was considered to be one of the mechanisms. Also, the ventricular effective refractory period (ERP) and the duration of monophasic action potential (MAPD) were prolonged, showing the "dome-formation" in phase 2 of the MAP trace [9].
It has been noted that the results of atrial biopsies taken from patients with AF have demonstrated the inflammatory infiltrates and oxidative damage within the atrial tissue [5]. In the study of Frustaci et al. abnormal atrial histology was found in multiple biopsy from patients with lone AF. The histological findings in biopsies taken from the interatrial septum were coincided with a diagnosis of myocarditis in $66 \%$ of patients [10]. Presence of circulating autoantibodies against heavy chains of myosin in a the vast majority of patients with idiopathic paroxysmal AF (PAF), raises the possibility of an inflammatory autoimmune processes [11].

\section{AF and inflammatory biomarkers}

AF is the most common sustained cardiac arrhythmia in clinical practice [12]. It is associated with an increased risk in mortality and morbidity, and may exert an adverse effect on the quality of life [13]. The pulmonary veins are the key trigger sites initiating AF. So far the exact stimulus for this focal triggering is unknown. Nevertheless, inflammation may provide one explanation. Indeed, inflammation can also act as an ongoing accelerator in the remodeling process [14]. The pathophysiology of AF is highly complex. It is usually associated with organic heart disease but it may also occur without clinically evident abnormalities. The main pathophysiological mechanisms contributing to the AF's development and progression include both electrical and structural remodeling of the atria. Moreover, AF itself can cause progressive electrophysiological changes during atrial remodeling, which perpetuates the arrhythmia-known as 'AF begets $A F^{\prime}$ phenomenon [15].

There have been many biomarkers associated with pathophysiology of AF such as C-reactive protein (CRP), tumor necrosis factor (TNF)- $\alpha$, interleukin (IL)-2, IL-6, IL-8, and monocyte chemoattractant protein (MCP)-1 [16]. Inflammatory biomarkers related to atrial fibrillation are listed in Table 1. The vascular markers that have been studied most frequently include high-sensitivity C-reactive protein (hs-CRP) and interleukin (IL)-6.

High sensitivity CRP is an acute-phase protein, factor known initially for its capacity to bind to the c-polysaccharide of Streptococcus pneumoniae. It has developed as the most robust and reproducible marker of vascular inflammation. CRP is synthesized primarily by the liver in response to pro-inflammatory cytokines. CRP is involved in the immune response, phagocytosis/opsonisation of the infectious agent and modulates the function of granulocytes and monocytes. Current- 
Table 1. Biomarkers of inflammation involved in the pathophysiology of atrial fibrillation $[5,16]$

\begin{tabular}{ll}
\hline $\begin{array}{l}\text { Inflammatory } \\
\text { Biomarkers }\end{array}$ & \multicolumn{1}{c}{ Targets and Effects } \\
\hline IL-2 & $\begin{array}{l}\text { Secreted by T cells stimulates growth and differentiation of T cell response. II-2 is also a predictor of AF after cardioversion } \\
\text { and after surgery. }\end{array}$ \\
\hline IL-6 & $\begin{array}{l}\text { Secreted by macrophages and endothelial cells influences adaptive immunity. IL-6 has both proinflammatory and cytoprotective } \\
\text { functions. It has been proven that IL-6 is also a predictor of AF after cardioversion and after ablation. Combined with CRP it } \\
\text { independently predicts strokes in patients with AF. }\end{array}$ \\
\hline IL-8 & Chemokine is produced by macrophages and epithelial cells associated with atrial fibrillation. \\
\hline hs-CRP & $\begin{array}{l}\text { Secreted by hepatocytes is a predictor of the development of AF after cardiac surgery and after ablation. Increased levels of CRP } \\
\text { have been found in patients with new-onset AF. Longer duration of AF is associated with higher CRP levels and larger LA diameter. }\end{array}$ \\
\hline MCP-1 & $\begin{array}{l}\text { One of the key chemokines that regulate migration and infiltration of monocytes/macrophages. Increased levels of MCP-1 have } \\
\text { been found in lone atrial fibrillation }\end{array}$ \\
\hline TNF- $\alpha$ & $\begin{array}{l}\text { Produced by macrophages is a cytokine involved in systemic inflammation. Increased levels of TNF- } \alpha \text { have been found in patients } \\
\text { with AF as opposed to individuals with sinus rhythm. In patients with chronic non-valvular AF it has been found that TNF- } \alpha \text { is } \\
\text { a predictor of ischemic stroke. TNF induces abnormal Ca }{ }^{2+} \text { handling and arrhythmogenicity in pulmonary vein cardiomyocytes. }\end{array}$ \\
\hline HSP27 & HSP-27 can downregulate TNF expression and upregulate IL-10 levels. HSP-27 is a predictor of AF recurrence after catheter ablation. \\
\hline
\end{tabular}

ly, there is a consistent and significant association, in all populations, between baseline hs-CRP levels and risk of future cardiovascular events like: (i) stroke, (ii) peripheral vascular disease, (iii) sudden cardiac death, (iv) $A F$, (v) plaque rupture, (vi) recurrent ischaemia, and (vii) myocardial infarction [17]. In patients with $\mathrm{AF}$, inflammatory cytokines IL- 6 and CRP were related to the higher risk of vascular death and thromboembolic events independent of clinical risk factors [18]. In another study the circulating biomarkers of inflammation, CRP, and oxidative stress, oxLDL, were higher in lone AF patients compared to healthy individuals and both biomarkers were associated with an increased risk of hypertension in lone AF [19].

The neutrophil/lymphocyte ratio (NLR) has been evaluated as a new predictor of cardiovascular risk, which is an inexpensive, easy to obtain, and widely available marker of inflammation. Additionally, it can be of use as the risk stratification marker in patients with various cardiovascular diseases. It could be used as an alternative to the traditional markers. For example, in one study, elevated pre- and postoperative N/L ratios were associated with an increased occurrence of AF after coronary artery bypass grafting [20]. Mesut Aydin et al. in retrospective study found significantly higher neutrophil/lymphocyte ratio (NLR) values in patients with documented supraventricular tachycardia (SVT) compared with their control group [21].

\section{AF and hypertension}

The relation between inflammation and AF in patients with hypertension has not yet been established [5]. So far, in the animal model of hypertension, leucocyte infiltration into the atria as well as inflammation followed by atrial fibrosis have been investigated [22]. It has been observed that the atrial stretch, due to the elevated left ventricular diastolic pressure in patients with hypertension, might activate regional renin-angiotensin-aldosterone system (RAAS), cardiac apoptosis, and oxidative stress, which can subsequently induce the regional inflammation in the heart [23]. Experimental studies have also revealed that angiotensin II possesses several pro-inflammatoryppoppentiëesB|\&tteking of the RAAS by either ACE-inhibitors or angiotensin II receptor blockers have shown to improve endothelial function and reduce inducibility of AF [24].

\section{Cardiosurgery and ablation}

AF remains one of the most frequent complications after coronary artery bypass graft surgery (CABG). Bruins et al. were first to propose the inflammation-AF hypothesis, following their observations of an increased frequency of AF after CABG [25]. After studying the activation of a complement system during and after $C A B G$, they reported the biphasic complement activation. The first phase occurs during cardiopulmonary bypass itself while the second phase occurs during the first 5 days after surgery, which involves CRP elevation associated with atrial arrhythmias. Interestingly, in the study of Ishida et al. which included patients undergoing off-pump CABG surgery, statistic analysis indicated that the highest quartile of IL-6 level immediately after the surgery varied by patients' age and can independently predict postoperative AF [26]. It was observed that nonsteroidal anti-inflammatory medications given in the imme- 
diate postoperative period of CABG surgery are relatively safe and effective in reducing the incidence of AF and favorably affect the length of hospitalization. In addition, Marcus et al. have proved that CRP and IL-6 levels were elevated in patients with atrial flutter (AFI). These levels of biomarkers fall significantly after ablation of $\mathrm{AFI}$, which proved that arrhythmia is the cause of the inflammation [27]. Indeed, Richter et. al showed that markers of oxidative stress and inflammation were up-regulated 2 days after ablation of atrial fibrillation and their up-regulation was associated with an early recurrence of AF, but it didn't predict long-term outcome [28].

In the study of Lim the increased levels of inflammatory biomarkers were observed within the first 3 days after radiofrequency (RF) ablation for AF. The level of inflammatory response was directly associated with early AF recurrence. The elevation in the prothrombotic markers, fibrinogen and D-dimer occurred 7 days after ablation and was correlated with the inflammatory response [29].

In another study which compared cryoablation and open-irrigated RF ablation for AFl, the increase in inflammatory markers was significantly greater in the second method. The higher CRP levels during the RF energy might have occurred due to endothelial damage and surface thrombosis. Patients who received cryoablation, had higher levels of myocardial injury (CK, CKMB, TnT) due to cardiomyocyte swelling and irreversible cell death by the rupture of membranes [30].

In the study of Malmborg et. al cryoballon cather ablation was also associated with greater myocardial injury than the one performed by RF energy-based pulmonary vein ablation catheter (PVAC). Nevertheles, there was no difference in the coagulation and inflammation activity between these two techniques [31].

\section{AF and electrical remodeling}

The key features of electrical remodeling are shortening of the atrial refractory period, the loss of rate adaptation, an increase in dispersion, slowing down of conduction velocity, and the presence of triggering foci. The shortening of the atrial effective refractory period takes place due to accumulation of calcium within atrial myocytes and, as a result, leads to the reduction of the inward L-type $\mathrm{Ca}^{2+}$ current, which in turn contributes to the promotion and maintenance of multiple wavelet-reentry circuits. Decreased Na current $\left(\mathrm{I}_{\mathrm{Na}}\right)$, impaired connexin channel function and enhanced fibrosis deposition, by contrast, slow conduction and promote AF-maintaining reentry [32]. Calcium homeostasis in cardiomyocytes is regulated by TNF, PDGF, and IL-2, which are associated with increased triggering and shortening of the action potential duration. Abnormal intracellular $\mathrm{Ca}^{2+}$ handling is emerging as a key contributor to AF that promotes both ectopic activity and reentry. Delayed afterdepolarization is initiated by diastolic $\mathrm{Ca}^{2+}$ leak from the sarcoplasmic reticulum $[5,32]$ According to Lee et. al, TNF induces abnormal $\mathrm{Ca}^{2+}$ handling and arrhythmogenicity in pulmonary vein cardiomyocytes [33]. TNF can also decrease the expression of sarcoplasmic/endoplasmic reticulum $\mathrm{Ca}^{2+}$ ATPase 2a (SERCA2a) by enhancing methylation in the promoter region [34]. PDGF-A, which promotes cell proliferation and collagen expression in cardiac fibroblasts, increases atrial fibrosis and susceptibility to AF [35]. In clinical studies, high CRP and IL-6 levels, are associated with increased atrial size, which further contributes to inflammation in atrial remodeling [36]. Indeed, the levels of myeloperoxidase in atrial tissue are higher in patients with $\mathrm{AF}$ than in individuals with sinus rhythm. Also, the increased blood myeloperoxidase level has been associated with early AF recurrence after catheter ablation [37].

\section{Ventricular arrhythmias, inflammation and the prevention of sudden cardiac death}

Approximately $50 \%$ of cardiac arrests occur in case of individuals without a known heart disease but most of them suffer from a concealed ischaemic heart disease. Nevertheless, it is known that inflammatory heart diseases lead to malignant ventricular arrhythmias and sudden cardiac death [38]. In nested, case-control study of apparently healthy male physicians, baseline plasma CRP levels were positively associated with risk of sudden cardiac death (SCD) over the ensuing 17 years of follow-up. The meantime to SCD was 9.2 years (range 0.7 to 16.8 years). One clinical implication of these data is that markers of inflammation may be useful in identifying men at higher risk of SCD who have no obvious signs of coronary heart disease (CHD) many years before the fatal event [39]. In another study of six hundred seventy eight healthy middle-aged and elderly subjects with no apparent heart disease and frequent ventricular premature complexes (VPC), a CRP values $\geqslant 2.5 \mu \mathrm{g} / \mathrm{mL}$ were associated with a significantly higher risk of death and acute myocardial infarction [40]. 


\section{Myocarditis}

Myocarditis is the pathological myocardial infection and autoimmunity that causes active inflammatory destruction of myocytes. Aetiologically, a wide spectrum of infectious agents, including viruses, bacteria as well as toxic and hypersensitivity reactions might be involved. Enteroviruses, especially Coxsackie B, adenoviruses and parvovirus B19 are among the most common causal agents. The typical microscopic image involves the presence of inflammatory cells together with necrotic myocytes [41].

Ventricular premature beats are typical forms of cardiac arrhythmias in myocarditis. Nevertheless, they may also include malignant ventricular tachycardia or ventricular fibrillation. Myocarditis can also be a factor responsible for sudden cardiac death in young people [42]. The electrocardiogram (ECG) is a sensitive tool used for diagnosis of myocarditis (in case of abnormal ST - T waves and conduction block are frequently observed in myocarditis). ECG manifestations are diverse, and include atrioventricular block (I to III degree), intraventricular conduction delay (widened QRS complex), frequent premature beats, supraventricular tachycardia, atrial fibrillation, sinus arrest, ventricular tachycardia, ventricular fibrillation, and asystole [43].

Considering malignant arrhythmias associated with myocarditis, two distinct clinical settings have to be distinguished: acute fulminant myocarditis and inflammatory cardiomyopathy. The first is associated with refractory malignant ventricular tachyarrhythmias in the context of severe acute heart failure (HF), and adverse short-term prognosis with early death due to multisystem failure. On the other hand long-term evolution to inflammatory cardiomyopathy with LV dysfunction results in a high risk of sudden cardiac death (SCD) similar to that for dilated cardiomyopathy (DCM). Patients with fulminant myocarditis have a high mortality rate and a severe risk of life-threatening refractory ventricular tachyarrhythmias. The important association between undiagnosed myocarditis and SCD is emphasized by post-mortem data, which have implicated myocarditis in SCD of young adults at rates of $8.6-44 \%$ [38].

In fulminant myocarditis most patients have markedly increased leucocytes (WBC) and CRP. Moreover in the study of Aoyama et al., all readmitted patients had abnormally increased WBC and CRP during their follow-up [44]. In another study creatine kinase (CK) and CRP levels declined sharply during the therapy of fulmi- nant myocarditis by intravenous methyloprednisolone, which was associated with hemodynamic improvement measured by the improved left ventricule ejection fraction (LVEF) [45].

In the study of Bironaite et al. the concentration of IL- 6 and hsCRP in the inflammatory dilated cardiomyopathy (iDCM) was increased. Persistent myocardial stressors increase T-lymphocytes in myocardium of iDCM patients. Moreover, chronic myocardial inflammation affected mitochondria and triggered significant release of Hsp-60, MMP-9/TIMP1 suggesting apoptotic pathways [46].

The pathogenesis of cardiac arrhythmias in the myocarditis is complex. The process was explained in the murine model of myocarditis. One of the triggers is the active process of inflammation related to the presence of the virus in the first stage of the disease. This process leads to the activation of immune system, characterized by the invasion of the natural killer cells and macrophages followed by T-lymphocytes. In this stage various cytokines including IL-1 $\beta$, TNF- $\alpha$, IFN- $\gamma$, and IL-2 are produced. The next process called subacute myocarditis is defined by activated virus-specific $T$ lymphocytes. These cells plays critical role in eliminating infected myocardial cells and limiting viral replication [47]. Pathological changes in the myocardium especially myocardial necrosis and replacement fibrosis (favoring re-entry mechanism) seem to be the major arrhythmogenic substrate. Proarrhythmic effects of the cytokines and inflammatory mediators may be related to the changes in the ion channel function (especially in the potassium and calcium channels). These modifications leads to the prolongation of the ventricular effective refractory period.

\section{Pericarditis}

The etiology of pericarditis may be similar to myocarditis with infective (viruses, bacteria) an non-infective (i.e. autoimmunity) agents. Acute pericarditis in isolation does not seem to be frequently associated with ventricular arrhythmia but is often present as perimyocarditis with a burden of arrhythmia related to the myocardial component [43]. Furthermore, SCD in these patients has mostly a haemodynamic and not an arrhythmic cause.

According to Imazio et al. C-reactive protein, WBC levels were higher in patients with pericarditis compared with those with myopericarditis/perimyocarditis [48]. In another study hs-CRP levels were elevated at the initial presentation in $78 \%$ of patients and com- 
prised the independent risk factors for recurrence of pericarditis. The higher CRP levels also may also support the clinical suspicion of pericarditis, but there is also a small percentage of patients with persistently negative hs-CRP which provides evidence that negative hs-CRP does not role out pericarditis [49]. There is also evidence that anti-inflammatory therapy is associated with the reduction in pericardial and systemic inflammation [50]. The levels of CRP in idiopathic recurrent acute pericarditis (IRAP) may be useful in monitoring the disease activity and choosing the appropriate length of therapy.

\section{Conclusion}

Inflammation plays an important role in the formation and maintenance of cardiac arrhythmias. Inflammatory biomarkers may prove useful in predicting the onset of cardiac arrhythmias and their complications. Inflammation and oxidative stress directly affect myocyte apoptosis and cardiac fibrosis, thus lead to progressive electrophysiological changes of the myocardium, which consequently contribute to arrhythmias. A better knowledge of the role of inflammation in the pathophysiology of cardiac arrhythmias is necessary to individualize treatment and develop future therapeutic strategies. New therapies might require the specific targeting of inflammatory biomarkers in addition to the modification of other factors to produce beneficial results [5]. Also, further clinical trials are necessary for the development of anti-inflammatory therapies in the prevention of AF. Identifying unique methods to improve imaging, autonomic modulation, inflammatory biomarkers will help us to better understand the patients with cardiac arrhythmias.

$$
\begin{array}{ll}
\text { List of abbreviations } \\
\text { AF } & \text { - atrial fibrillation } \\
\text { PAF } & \text { - paroxysmal atrial fibrillation } \\
\text { CRP } & \text { - C-reactive protein } \\
\text { IL } & \text { - interleukin } \\
\text { TNF } & \text { - Tumor necrosis factor } \\
\text { MCP } & \text { - Monocyte chemoattractant protein } \\
\text { EAM } & \text { - experimental autoimmune myocarditis } \\
\text { NLR } & \text { - neutrophil/lymphocyte ratio } \\
\text { SVT } & \text { - supraventricular tachycardia } \\
\text { RAAS } & \text { - renin-angiotensin-aldosterone system } \\
\text { CABG } & \text { - coronary artery bypass graft } \\
\text { AFI } & \text { - atrial flutter } \\
\text { RF } & \text { - radiofrequency } \\
\text { SCD } & \text { - sudden cardiac death }
\end{array}
$$

$$
\begin{aligned}
& \text { CHD - coronary heart disease } \\
& \text { VPC - ventricular premature complexes } \\
& \text { ECG - electrocardiogram } \\
& \text { HF - heart failure } \\
& \text { DCM - dilated cardiomyopathy } \\
& \text { IRAP - idiopathic recurrent acute pericarditis } \\
& \text { MAPD - monophasic action potential duration } \\
& \text { ERP - effective refractory period }
\end{aligned}
$$

\section{Acknowledgements}

\section{Conflict of interest statement}

The authors declare no conflict of interest.

\section{Funding sources}

There are no sources of funding to declare.

\section{References}

1. Gregor MF, Hotamisligil GS. Inflammatory mechanisms in obesity. Annu Rev Immunol. 2011;29:415-445.

2. Libby P. Inflammation and cardiovascular disease mechanisms. The American journal of clinical nutrition. 2006;83(2):456-460.

3. Lip GY, Patel JV, Hughes E, Hart RG. High-sensitivity C-reactive protein and soluble CD40 ligand as indices of inflammation and platelet activation in 880 patients with nonvalvular atrial fibrillation: relationship to stroke risk factors, stroke risk stratification schema, and prognosis. Stroke. 2007;38:1229-1237.

4. Feuerstein GZ, Libby P, Mann DL. Inflammation-a new frontier in cardiac disease and therapeutics. Inflammation and Cardiac Diseases. Birkhäuser Basel. 2003; 1-5.

5. Hu YF, Chen YJ, Lin YJ, Chen SA. Inflammation and the pathogenesis of atrial fibrillation. Nature Reviews Cardiology. 2015;12(4):230-243.

6. Pasquie JL, Sanders $P$, Hocini $M$ et al. Fever as a precipitant of idiopathic ventricular fibrillation in patients with normal hearts. J Cardiovasc Electrophysiol. 2004;15:1-6.

7. Klein RM,Vester EG, Brehm MU et al. Inflammation of the myocardium as an arrhythmia trigger. Zeitschrift fur Kardiologie. 1999;89:24-35.

8. Pieroni M, Smaldone C, Bellocci F. Myocarditis presenting with ventricular arrhythmias: role of electroanatomical mapping-guided endomyocardial biopsy in differential diagnosis. In Cihakova D, ed. Myocarditis. InTech Open Access Publisher. 2011:365-386.

9. Saito, Junko, Niwano S, Niwano H et al. Electrical remodeling of the ventricular myocardium in myocarditis. Circulation Journal. 2002;66(1):97-103.

10. Frustaci A, Chimenti C, Bellocci F, Morgante E, Russo MA, Maseri A. Histological substrate of atrial biopsies in patients with lone atrial fibrillation. Circulation. 1997;96:1180-1184.

11. Maixent JM, Paganelli F, Scaglione J, Levy S. Antibodies against myosin in sera of patients with idiopathic paroxysmal atrial fibrillation. Journal of cardiovascular electrophysiology. 1998;9(6):612-617.

12. Peters NS, Schilling RJ, Kanagaratnam $P$, Markides V. Atrial fibrillation: strategies to control, combat, and cure. Lancet. 2002;359:593-603. 
13. Gronefeld GC, Lilienthal J, Kuck KH, Hohnloser SH; Impact of rate versus rhythm control on quality of life in patients with persistent atrial fibrillation: results from a prospective randomized study. Eur Heart J. 2003;24:1430-1436.

14. Haissaguerre M, Marcus FI, Fischer B, Clementy J. Radiofrequency catheter ablation in unusual mechanisms of atrial fibrillation. J Cardiovasc Electrophysiol. 1994;5:743-751.

15. Wijffels MC, Kirchhof CJ, Dorland R, Allessie MA. Atrial fibrillation begets atrial fibrillation: a study in awake chronically instrumented goats. Circulation. 1995;92:1954-1968.

16. Li J, Solus J, Chen $Q$ et al. Role of inflammation and oxidative stress in atrial fibrillation. Heart Rhythm. 2010;7(4):438444.

17. Blake GJ, Ridker PM. C-reactive protein and other inflammatory risk markers in acute coronary syndromes. J Am Coll Cardiol. 2003;41(Suppl. 4):37-42.

18. Aulin J et al. Interleukin- 6 and C-reactive protein and risk for death and cardiovascular events in patients with atrial fibrillation. American Heart Journal. 2015;170(6):11511160.

19. Polovina MM, Ostojic MC, Potpara TS. Relation of biomarkers of inflammation and oxidative stress with hypertension occurrence in lone atrial fibrillation. Mediators of inflammation. 2015. Doi: 10.1155/2015/653026.

20. Gibson PH, Cuthbertson BH, Croal BL et al. Usefulness of neutrophil/lymphocyte ratio as predictor of new-onset atrial fibrillation after coronary artery bypass grafting. The American Journal of Cardiology. 2010;105(2):186-191.

21. Aydın M, Yıldız A, Yüksel M et al. Assessment of the neutrophil/lymphocyte ratio in patients with supraventricular tachycardia. Anatolian journal of cardiology. 2015; 15(0):000-000

22. Lau DH, Mackenzie L, Kelly DJ et al. Hypertension and atrial fibrillation: evidence of progressive atrial remodeling with electrostructural correlate in a conscious chronically instrumented ovine model. Heart Rhythm. 2010;7: 1282-1290.

23. De Jong AM, Maass AH, Oberdorf-Maass SU, Van Veldhuisen DJ, Van Gilst WH, Van Gelder IC, et al. Mechanisms of atrial structural changes caused by stretch occurring before and during early atrial fibrillation. Cardiovasc Res. 2011;89:754-765.

24. Boos CJ, Anderson RA, Lip GY. Novel therapies for the prevention of stroke. Expert Opin Investig Drugs, 2004;13:1615-1630.

25. Bruins $P$, te Velthuis $H$, Yazdanbakhsh AP et al. Activation of the complement system during and after cardiopulmonary bypass surgery postsurgery activation involves $\mathrm{C}$-reactive protein and is associated with postoperative arrhythmia. Circulation. 1997;96(10):3542-3548.

26. Ishida K, Kimura F, Imamaki M et al. Relation of inflammatory cytokines to atrial fibrillation after off-pump coronary artery bypass grafting. European Journal of Cardio-Thoracic Surgery. 2006;29(4):501-505.

27. Marcus GM, Smith LM, Glidden DV et al. Markers of inflammation before and after curative ablation of atrial flutter. Heart Rhythm. 2008;5(2):215-221.

28. Richter B, Gwechenberger M, SocasA, et al. Markers of oxidative stress after ablation of atrial fibrillation are associated with inflammation, delivered radiofrequency energy and early recurrence of atrial fibrillation. Clinical Research in Cardiology. 2012;101(3):217-225.
29. Lim HS, Schultz C, Dang J et al. Time course of inflammation, myocardial injury, and prothrombotic response after radiofrequency catheter ablation for atrial fibrillation. Circulation: Arrhythmia and Electrophysiology. 2014;7(1):83-89.

30. Oswald H, Gardiwal A, Lissel C et al. Difference in humoral biomarkers for myocardial injury and inflammation in radiofrequency ablation versus cryoablation. Pacing and clinical electrophysiology. 2007;30(7):885-890

31. Malmborg H, Christersson C, Lönnerholm S, Blomström-Lundqvist $C$ et al. Comparison of effects on coagulation and inflammatory markers using a duty-cycled bipolar and unipolar radiofrequency pulmonary vein ablation catheter vs. a cryoballoon catheter for pulmonary vein isolation. Europace. 2013;15(6):798-804.

32. Dobrev D, Carlsson L, Nattel S. Novel molecular targets for atrial fibrillation therapy. Nature Reviews Drug Discovery. 2012;11(4):275-291.

33. Lee SH, Chen YC, Chen YJ et al. Tumor necrosis factor- $\alpha$ alters calcium handling and increases arrhythmogenesis of pulmonary vein cardiomyocytes. Life Sci. 2007;80:1806-1815.

34. Kao YH, Chen YC, Cheng CC et al. Tumor necrosis factor- $\alpha$ decreases sarcoplasmic reticulum Ca2+-ATPase expressions via the promoter methylation in cardiomyocytes. Crit. Care Med. 2010;38:217-222

35. Liao $\mathrm{CH}$, Akazawa $\mathrm{H}$, Tamagawa $\mathrm{M}$ et al. Cardiac mast cells cause atrial fibrillation through PDGF-A-mediated fibrosis in pressure-overloaded mouse hearts. J Clin Invest. 2010;120:242-253.

36. Psychari SN, Apostolou TS, Sinos L, Hamodraka E, Liakos G, Kremastinos DT. Relation of elevated C-reactive protein and interleukin- 6 levels to left atrial size and duration of episodes in patients with atrial fibrillation. The American journal of cardiology. 2005;95(6):764-767

37. Li SB, Yang F, Jing L et al. Myeloperoxidase and risk of recurrence of atrial fibrillation after catheter ablation. J Investig Med. 2013;61:722-727.

38. Priori SG, Blomström-Lundqvist C, Mazzanti A et al. 2015 ESC Guidelines for the management of patients with ventricular arrhythmias and the prevention of sudden cardiac death. Europace. 2015: euv319.

39. Albert CM, Ma J, Rifai N, Stampfer MJ, Ridker PM. Prospective study of C-reactive protein, homocysteine, and plasma lipid levels as predictors of sudden cardiac death. Circulation. 2002;105:2595-2599.

40. Sajadieh A, Nielsen OW, Rasmussen V, Ole Hein H, Hansen JF. Increased ventricular ectopic activity in relation to C-reactive protein, and NT-pro-brain natriuretic peptide in subjects with no apparent heart disease. Pacing Clin Electrophysiol. 2006;29:1188-1194.

41. Sagar S, Liu PP, Cooper LT. Myocarditis. The Lancet. 2012;379(9817):738-747.

42. Baksi, AJ, Kanaganayagam GS, Prasad SK. Arrhythmias in Viral Myocarditis and Pericarditis. Cardiac electrophysiology clinics. 2015;7(2):269-281.

43. JCS Joint Working Group. Guidelines for diagnosis and treatment of myocarditis (JCS 2009): digest version. Circulation journal: official journal of the Japanese Circulation Society. 2011;75(3):734. 
44. Aoyama N, Izumi T, Hiramori K, et al. National survey of fulminant myocarditis in Japan. Circulation Journal. 2002;66(2):133-144.

45. Nakashima H, Umeyama Y, Minami K. Successive immunosuppressive treatment of fulminant myocarditis that is refractory to mechanical circulatory support. Medical Science Monitor Basic Research. 2013;14:116-119.

46. Bironaite D, Daunoravicius D, Bogomolovas J, et al. Molecular mechanisms behind progressing chronic inflammatory dilated cardiomyopathy. BMC cardiovascular disorders. 2015;15(1):1.

47. Kawai Ch. From myocarditis to cardiomyopathy: mechanisms of inflammation and cell death learning from the past for the future. Circulation. 1999;99(8):1091-1100.

48. Imazio M, Brucato A, Barbieri A et al. Good prognosis for pericarditis with and without myocardial involvement: results from a multicenter prospective cohort study. Circulation (2013): CIRCULATIONAHA-113.

49. Imazio M, Brucato A, Maestroni $S$ et al. Prevalence of C-Reactive Protein Elevation and Time Course of Normalization in Acute Pericarditis Implications for the Diagnosis, Therapy, and Prognosis of Pericarditis. Circulation. 2011;123(10):1092-1097.
50. Feng D, Glockner J, Kim K et al. Cardiac Magnetic Resonance Imaging Pericardial Late Gadolinium Enhancement and Elevated Inflammatory Markers Can Predict the Reversibility of Constrictive Pericarditis After Antiinflammatory Medical Therapy A Pilot Study. Circulation. 2011;124(17):1830-1837.

Acceptance for editing: 2016-06-10 Acceptance for publication: 2016-06-23

\section{Correspondence address:}

Jan Budzianowski MD

Department of Cardiology, Hospital Nowa Sól

7 Chałubińskiego Street, 67-100 Nowa Sól, Poland phone: +48695255829 fax: +4868 3877003 email: jan.budzianowski@gmail.com 